\begin{tabular}{|c|c|c|c|}
\hline \multirow{3}{*}{$\begin{array}{r}\text { Case Reports in } \\
\text { Gastroenterology }\end{array}$} & Case Rep Gastroenterol 20 & & \multirow[b]{2}{*}{$\begin{array}{l}\text { Karger } \\
\text { Open access }\end{array}$} \\
\hline & $\begin{array}{l}\text { DOI: } 10.1159 / 000448989 \\
\text { Publisnea onine: Uctover 6, } 2016\end{array}$ & $\begin{array}{l}\text { ( } 2016 \text { The Author(s) } \\
\text { Published by S. Karger AG, Basel } \\
\text { www.karger.com/crg }\end{array}$ & \\
\hline & $\begin{array}{l}\text { This article is licensed under th } \\
\text { International License (CC BY-NC) } \\
\text { Usage and distribution for commer }\end{array}$ & $\begin{array}{l}\text { nons Attribution-NonCommercial } 4 \\
\text { ger.com/Services/OpenAccessLicense } \\
\text { uires written permission. }\end{array}$ & \\
\hline
\end{tabular}

\title{
Isolated Liver Hilar Infiltration by IgG4 Inflammation Mimicking Cholangiocarcinoma
}

\author{
Laurent Bochatay Pietro Majno Emiliano Giostra Jean Louis Frossard
}

Geneva University Hospitals, Geneva, Switzerland

\section{Keywords}

IgG4 liver disease $\cdot$ Cholangiocarcinoma $\cdot$ Hilar filling defect

\begin{abstract}
IgG4-related disease represents a heterogeneous group of disease characterized by infiltration of various tissues by IgG4 plasmocytes. In case of liver infiltration, this condition classically mimics primary sclerosing cholangitis or multifocal cholangiocarcinoma due to inflammation that preferentially affects the intra- and extrahepatic bile duct. Diagnostic criteria have recently been reviewed in order to better define the disease and help physicians make the diagnosis. Herein, we present the case of a patient who died after liver surgery for suspected cholangiocarcinoma that finally turned out to be IgG4-associated liver disease, a condition being out of current consensual criteria. The patient presented with progressive cholestasis identified by MR cholangiography as an isolated hilar mass responsible for dilatation of the left and right intrahepatic bile duct suspicious for a Klatskin tumor. The IgG4 blood level was normal as was biliary cytology. The patient underwent right portal embolization followed by right extended hepatectomy. Pathologic examination found no tumor but intense fibrosclerotic infiltration with a marked inflammatory infiltrate characterized by IgG4positive plasmocytes. Despite immunosuppressive treatment, cholestasis was never controlled and successive biopsies of the remaining liver showed progressive cholestasis, liver infiltrate and no bile duct regeneration. The patient finally presented an upper gastrointestinal hemorrhage leading to death 4 months after hepatectomy and appropriate immunosuppressive therapy.

(C) 2016 The Author(s)

Published by S. Karger AG, Basel
\end{abstract}




\section{Introduction}

IgG4-mediated disease is a multisystemic condition and is characterized by the infiltration of various tissues by IgG4-positive plasmocytes. The salivary glands, retroperitoneum, retroocular tissue, pancreas and hepatobiliary system can all be affected $[1,2]$. Type 1 autoimmune pancreatitis is the most studied disease but is still rare [3]. The hepatobiliary system is involved in $60-80 \%$ of the patients with type 1 autoimmune pancreatitis [4-6], most of the time mimicking primary sclerosing cholangitis in cholangiographic studies. Different classification systems have been proposed so far, but an international pathological consensus has been finally reached in 2012 [7], defining minimal conditions to diagnose IgG4related disease (table 1). Furthermore, a classification of cholangiography features in IgG4related sclerosing cholangitis has been proposed to help classify four patterns affecting the biliary tract [6]. Diagnosis and imaging criteria are aimed at helping clinicians to distinguish between IgG4 hepatobiliary disease and alternative diagnosis such as primary sclerosing cholangitis, pancreatic cancer, chronic pancreatitis, gallbladder and bile duct cancer.

We here present the case of a patient who died after liver surgery for suspected cholangiocarcinoma that finally revealed as IgG4 liver disease being out of consensual criteria.

\section{Case Presentation}

A 66-year-old male was admitted to our hospital because of persistent diarrhea, discolored stools, dark urine and weight loss. The liver tests showed significant cholestasis [alkaline phosphatase 4 times the upper normal limits (UNL), gamma-glutamyltransferase 20 times UNL and total bilirubin 1.2 times UNL], while MRI showed a thickening of the biliary convergence and the initial part of the left bile duct without surrounding infiltration. The intrahepatic biliary tree was slightly dilated. 3D reconstruction showed a hilar filling defect of the biliary tree (fig. 1). The lesion was highly suspicious for a Klatskin tumor, and the patient underwent percutaneous drainage with sampling, while an internal-external drain was left in place. Carbohydrate antigen $19-9$ was $16 \mathrm{kU} / \mathrm{l}$ (normal <37) and carcinoembryonic antigen was $2.2 \mu \mathrm{g} / \mathrm{l}$ (normal <4.6). Total immunoglobulin was IgG $13 \mathrm{mg} / \mathrm{l}, \mathrm{IgA} 2.83 \mathrm{~g} / \mathrm{l}$ and $\operatorname{IgM} 1.14 \mathrm{~g} / \mathrm{l}$ ), while the subclass IgG4 was $1.3 \mathrm{~g} / \mathrm{l}$. The ratio IgG4/IgG was $9 \%$. Biliary cytology was negative. Because of the strong suspicion of Klatskin tumor, the patient had a right portal embolization extended to segment IV in order to anticipate an extended right hepatectomy including segment IV, later allowing a right hepatectomy extended to segments I and IV with hilar lymphadenectomy.

Pathologic examination identified intense fibrosclerotic infiltration of the left and right biliary tract with marked inflammatory infiltrate characterized by IgG4 plasmocytes and an important amount of polymorphonuclear eosinophils. Circumferential 'peel of onion'-like fibrosis was also observed. There were no signs of malignancy. Plasmocytes were stained positive for IgG4. The surrounding liver parenchyma was normal.

The diagnosis of IgG4-related hepatobiliary disease was finally made, and prednisone was started. The postoperative course was complicated by a progressive increase of cholestasis parameters within the next 4 weeks (fig. 2). Liver biopsy identified major biliary duct dystrophia with IgG4 infiltration, explaining the progressive cholestasis. Treatment was reinforced with a combination of azathioprine and prednisone, allowing a slight improvement of the liver tests and patient discharge. 
One week later, the patient was readmitted with worsening jaundice. A CT scan excluded bile duct stenosis, while liver biopsy still showed a severe bile duct dystrophia. However, IgG4 plasmocytes had disappeared, and significant bilirubinostasis accumulating within the bile duct, portal space as well as inside the hepatocytes was observed. There were no ductular proliferative reactions.

The patient presented duodenal ulcer bleeding (Forrest Ia), leading to hemorrhagic shock and finally death 4 months after the right hepatectomy (fig. 2). The patient's autopsy demonstrated several liver damages that could explain the abnormal liver tests and function (prothrombin time 62\%, factor V 65\%). Liver autopsy showed liver atrophy and inadequate liver regeneration. The biliary dystrophies were identical with those already demonstrated by previous liver biopsies. Massive upper gastrointestinal hemorrhage was responsible for the immediate death of the patient.

\section{Discussion}

IgG4-related biliary disease induces most frequently stenosis and dilation of isolated or multiple branches of the biliary tree, mimicking primary sclerosing cholangitis or cholangiocarcinoma. International guidelines recommend against fine-needle punction or aspiration for the diagnosis of cholangiocarcinoma as long as it is considered surgically curable because of the risk of seeding cancer cells along the needle tract [7]. Serum dosage of immunoglobulin subtype, looking for IgG4 and brush cytology can help excluding IgG4-related disease before proceeding to surgery and is part of the diagnostic criteria $[8,9]$. The current case illustrates the fact that IgG4-related disease remains difficult to diagnose before surgery. As soon as the liver explant was analyzed by the pathologist, showing no tumor cells but massive plasmocytes IgG4-positive associated with bile ducts injuries, the diagnosis became evident.

Although prednisone was soon introduced, the patient's condition worsened, justifying combined azathioprine and prednisone treatment. Successive liver biopsies showed massive destruction of the small bile duct, explaining the progressive worsening of the cholestasis. Prednisone was probably at least partly effective as IgG4-positive plasmocytes infiltrated in the hepatic tissue disappeared between the day of surgery and the 2 successive liver biopsies. However, severe biliary dystrophy with the lack of regeneration of the small bile ducts observed in the second biopsy might explain the absence of improvement of the patient's liver tests. Finally, a fatal hemorrhage occurred.

Misdiagnosed type 1 pancreatitis leads to surgery for suspected pancreatic adenocarcinoma in many cases, as described in the literature [1]. Hopefully, this situation is much less common for IgG4-mediated biliary tract involvement. Case reports and few series of IgG4related sclerosing cholangitis of patients undergoing surgery are described in the literature [10-12], but our case was different to some extent. First, sclerosing cholangitis was not suspected because of an intact intrahepatic and extrahepatic bile duct apart from the hilar stenosis. Indeed, the absence of multiple biliary stenosis, a normal serum IgG and IgG4 levels and a normal IgG4/IgG ratio made the putative diagnosis of hilar cholangiocarcinoma most likely. Portal embolization, hepatic radical surgery and delay in prednisone treatment may have led to disease progression. The lack of hepatic reserve following large hepatic resection may have altered bile duct regeneration and promoted hepatic failure (prothrombin time $62 \%$, factor V 65\%). Finally, prednisone, physical stress following repeated hospitalizations as well as major surgery led to duodenal ulcers and fatal hemorrhage. 
Bochatay et al.: Isolated Liver Hilar Infiltration by IgG4 Inflammation Mimicking Cholangiocarcinoma

\section{Conclusion}

The diagnosis of IgG4-related biliary disease remains difficult because a blood test to rule out such diagnosis does not exist. The recourse to more invasive diagnostic tools such as percutaneous or endoscopic drainage allowing bile duct sampling is therefore often required. When surgically curable cholangiocarcinoma is the most likely diagnosis, tissue sampling is not a diagnostic option. IgG4-related bile duct isolated stenosis is rare, but this specific diagnosis should be kept in mind because consequences can be fatal, as illustrated in this case.

\section{Statement of Ethics}

The authors have no ethical conflicts to disclose.

\section{Disclosure Statement}

All authors declare that they have no conflicts of interest relevant to this paper.

\section{References}

1 Kazuich 0, Masahito Y, Toshiyuki M, Kazushige U: Recent advances in the concept and pathogenesis of IgG4-related disease in the hepato-bilio-pancreatic system. Gut Liver 2014;8:462-470.

2 Lang D, Zwerina J, Pieringer H: IgG4-related disease: current challenges and future prospects. Ther Clin Risk Manag 2016;12:189-199.

-3 Inoue D, Yoshida K, Yoneda N, et al: IgG4-related disease: dataset of 235 consecutive patients. Medicine (Baltimore) 2015;94:e680.

4 Okazaki K, Uchida K, Koyabu M, Miyoshi H, Takaoka M: Recent advances in the concept and diagnosis of autoimmune pancreatitis and IgG4-related disease. J Gastroenterol 2011;46:277-288.

5 Okazaki K, Uchida K, Matsushita M, Takaoka M: How to diagnose autoimmune pancreatitis by the revised Japanese clinical criteria. J Gastroenterol 2007;42(suppl 18):32-38.

6 Okazaki K, Kawa S, Kamisawa T, et al: Amendment of the Japanese consensus guidelines for autoimmune pancreatitis, 2013 I. Concept and diagnosis of autoimmune pancreatitis. J Gastroenterol 2014;49:567-588.

7 Khan SA, Davidson BR, Goldin RD, et al: Guidelines for the diagnosis and treatment of cholangiocarcinoma: an update. Gut 2012;61:1657-1669.

8 Ohara H, Okazaki K, Tsubouchi H, et al: Clinical diagnostic criteria of IgG4-related sclerosing cholangitis 2012. J Hepatobiliary Pancreat Sci 2012;19:536-542.

-9 Deshande V, Zen Y, Chan JK, et al: Consensus statement on the pathology of IgG4-related disease. Mod Pathol 2012;25:1181-1192.

10 Maeda M, Shimada K: A case of IgG4-related sclerosing cholangitis mimicking an intrahepatic cholangiocellular carcinoma. Jpn J Clin Oncol 2012;42:153.

11 Erdogan D, Kloek JJ, ten Kate FJ, Rauws EA, Busch OR, Gouma DJ, et al: Immunoglobulin G4-related sclerosing cholangitis in patients resected for presumed maliganant bile duct strictures. Br J Surg 2008;95:727-734.

12 Miki A, Sakuma Y, Ohzawa H, Sanada Y, Sasanuma H, Lefor A T, Sata N, Yasuda Y: Immunoglobulin G4related sclerosing cholangitis mimicking hilar cholangiocarcinoma diagnosed with following bile duct resection: report of a case. Int Surg 2015;100:480-485. 
Bochatay et al.: Isolated Liver Hilar Infiltration by IgG4 Inflammation Mimicking Cholangiocarcinoma

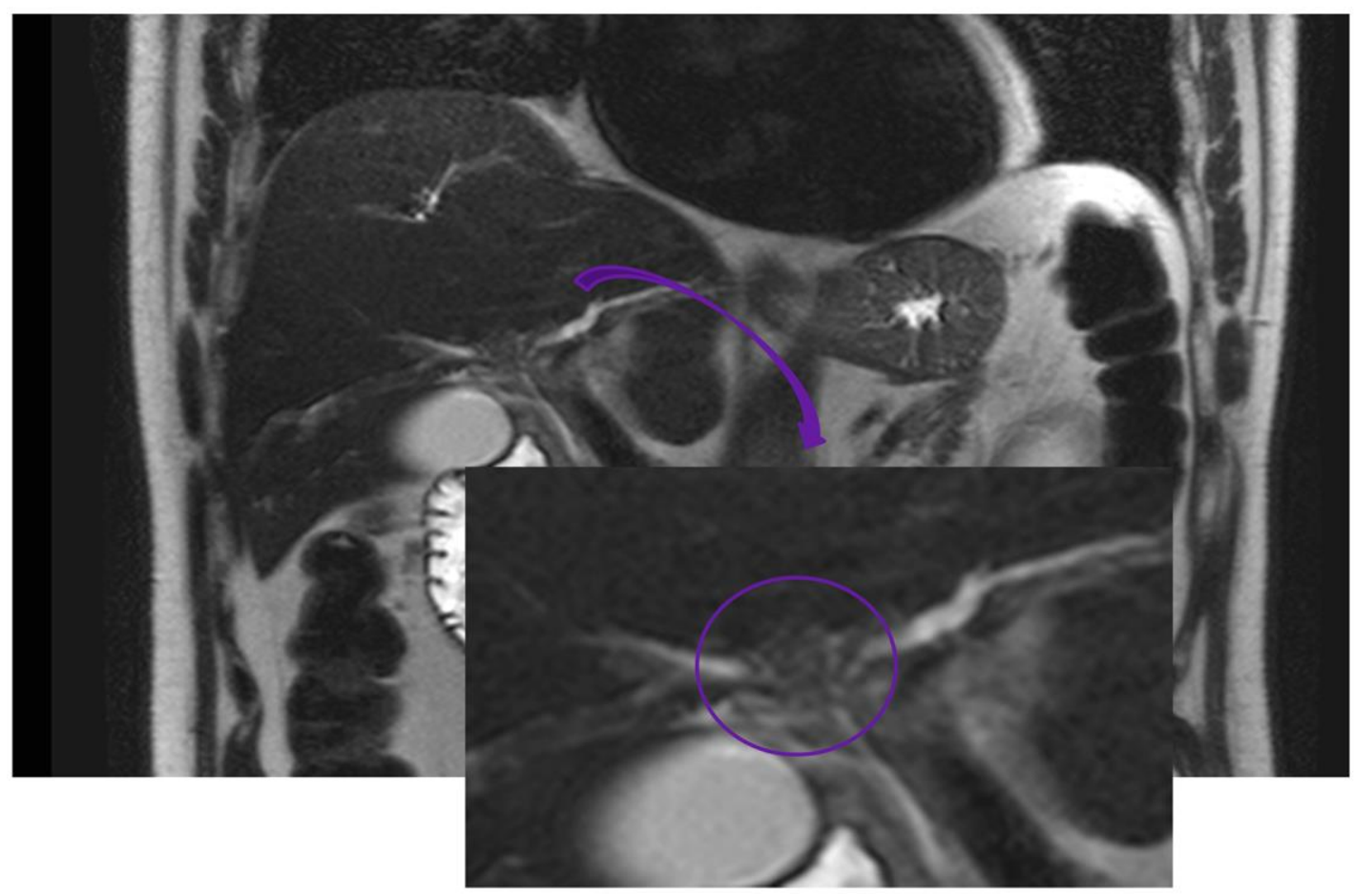

Fig. 1. MRI 3D reconstruction of the biliary tree showing central hilar filling defect. The arrow indicates magnification of the hilar region. The circle underlines a tissue-like structure responsible for the filling defect of the biliary convergence.

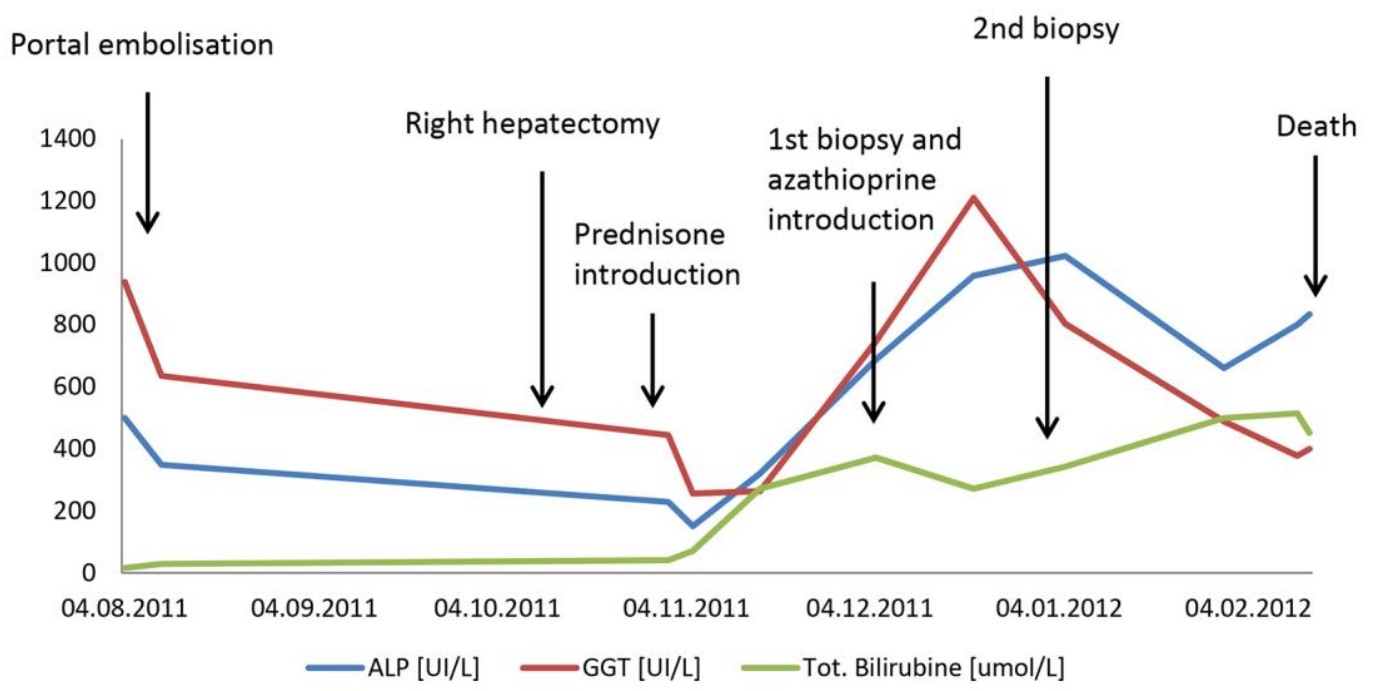

Fig. 2. Clinical course illustrated by cholestasis chemistry. Modifications in cholestasis chemistry were observed in major clinical events, indicated by arrows, from the time of hospitalization until the occurrence of death. APL = Alkaline phosphatase; GGT = gamma-glutamyltransferase. 


\section{Case Reports in Gastroenterology}

Case Rep Gastroenterol 2016;10:512-517

(c) 2016 The Author(s). Published by S. Karger AG, Basel www.karger.com/crg

Bochatay et al.: Isolated Liver Hilar Infiltration by IgG4 Inflammation Mimicking Cholangiocarcinoma

Table 1. 2012 international pathological consensus defining minimal conditions to diagnose IgG4-related disease

Major histopathological features associated with IgG4-related disease

1. Dense lymphoplasmacytic infitrate

2. Fibrosis, arranged at least focally in a storiform pattern

3. Obliterative phlebitis

Other histolpathological features associated with IgG4-related disease

1. Phlebitis without obliteration of the lumen

2. Increased number of eosinophils

Minimal criteria for IgG4-related disease in/at a new organ/site

1. Characteristic histopathological finding with an elevated IgG4t plasma cells and IgG4-to-IgG ratio

2. High serum IgG4 concentrations

3. Effective response to glucocorticoid therapy

4. Reports of other organ involvement that is consistent with IgG4-related disease 